\title{
PROXIMAL MONOTONICITY IN PARTIALLY ORDERED METRIC SPACES AND $F$-CYCLIC CONTRACTIONS
}

\author{
M. GABELEH, O. OLELA OTAFUDU, AND R. PLEBANIAK \\ Received 04 November, 2016
}

\section{INTRODUCTION AND PRELIMINARIES}

Recently, D. Wardowski ([7]) provided a new class of contractive mappings as below.

Definition 1. Let $\mathcal{F}$ be the collection of all functions $F: \mathbb{R}_{+} \rightarrow \mathbb{R}$ which satisfy the following conditions:

$(F 1) F$ is strictly increasing.

(F2) For each sequence $\left\{\alpha_{n}\right\}_{n \in \mathbb{N}}$ of positive numbers $\alpha_{n} \rightarrow 0 \Leftrightarrow F\left(\alpha_{n}\right) \rightarrow-\infty$.

(F3) There exists $k \in(0,1)$ such that $\lim _{\alpha \rightarrow 0^{+}} \alpha^{k} F(\alpha)=0$.

For a given metric space $(X, d)$, a mapping $T: X \rightarrow X$ is said to be an $F$ - contraction if there exists $\tau>0$ such that

$$
d(T x, T y)>0 \Rightarrow \tau+F(d(T x, T y)) \leq F(d(x, y)), \quad \forall x, y \in X .
$$

It is remarkable to note that every $F$ - contraction self-mapping is continuous. The following result is an extension of Banach contraction principe.

Theorem 1 ([7]). Let $(X, d)$ be a complete metric space and let $T: X \rightarrow X$ be an $F$-contraction. Then $T$ has a unique fixed point $x^{*} \in X$ and for every $x_{0} \in X$ the sequence $\left\{T^{n} x_{0}\right\}_{n \in \mathbb{N}}$ is convergent to $x^{*}$.

Another generalization of Banach contraction principe was established in [4] as follows.

Theorem 2. Let $A$ and $B$ be two nonempty closed subsets of a complete metric space $(X, d)$. Suppose that $T: A \cup B \rightarrow A \cup B$ is a cyclic mapping, that is $T(A) \subseteq$ $B$ and $T(B) \subseteq A$, such that $d(T x, T y) \leq \alpha d(x, y)$ for some $\alpha \in(0,1)$ and for all $x \in A, y \in B$. Then $T$ has a unique fixed point in $A \cap B$.

An interesting feature about the above observation is that the mapping $T$ in Theorem 2 may not be continuous.

It might be interesting to ask if in Theorem 1.1 $A \cap B=\varnothing$, then what happens. It is clear that the fixed point equation $T x=x$ has no fixed point. In this case, we have the following notion. 
Definition 2. Let $A, B$ be two nonempty subsets of a metric space $(X, d)$ and $T: A \cup B \rightarrow A \cup B$ be a cyclic mapping. A point $p \in A \cup B$ is called a best proximity point of $T$ if $d(p, T p)=\operatorname{dist}(A, B)$, where $\operatorname{dist}(A, B):=\inf \{d(x, y): x \in A, y \in B\}$.

Indeed best proximity point theorems have been studied to find necessary conditions such that the minimization problem

$$
\min _{x \in A \cup B} d(x, T x),
$$

has at least one solution, where $T$ is a cyclic mapping defined on $A \cup B$.

Let $A$ and $B$ be two nonempty subsets of a metric space $(X, d)$. We will use the following notations in our coming discussion.

$$
\begin{gathered}
d^{*}(x, y)=d(x, y)-\operatorname{dist}(A, B), \quad \forall(x, y) \in A \times B, \\
d(x, A)=\operatorname{dist}(\{x\}, A), \quad \forall x \in X, \\
\mathcal{P}_{A}(z)=\{x \in A: d(x, z)=d(z, A)\}, \quad \forall z \in X, \\
A_{0}:=\{x \in A: d(x, y)=\operatorname{dist}(A, B) \quad \text { for some } \quad y \in B\}, \\
B_{0}:=\{y \in B: d(x, y)=\operatorname{dist}(A, B) \quad \text { for some } \quad x \in A\} .
\end{gathered}
$$

Note that if $(A, B)$ is a nonempty, weakly compact and convex pair of subsets of a Banach space $X$, then $A_{0}$ and $B_{0}$ are also nonempty, closed and convex subsets of $X$.

Definition 3. Let $A$ and $B$ be nonempty subsets of a metric space $(X, d)$. We say that $A$ is Chebyshev set w.r.t. $B$ provided that $\mathcal{P}_{A}(x)$ is singleton for any $x \in B$.

For instance, if $A$ and $B$ are two nonempty, weakly compact and convex sets in a strictly convex Banach space $X$, then $A$ is Chebyshev set w.r.t. $B$ and $B$ is also Chebyshev set w.r.t. $A$.

In [6] Suzuki et al. introduced a notion of property UC on metric spaces as follows.

Definition 4. ([6]) Let $A$ and $B$ be nonempty subsets of a metric space $(X, d)$. Then $(A, B)$ is said to satisfy the property UC provided if $\left\{x_{n}\right\}$ and $\left\{z_{n}\right\}$ are sequences in $A$ and $\left\{y_{n}\right\}$ is a sequence in $B$ such that $\lim _{n} d\left(x_{n}, y_{n}\right)=\operatorname{dist}(A, B)$ and $\lim _{n} d\left(z_{n}, y_{n}\right)=\operatorname{dist}(A, B)$, then $\lim _{n} d\left(x_{n}, z_{n}\right)=0$.

Example 1.1.([2]) Let $A$ and $B$ be nonempty subsets of a uniformly convex Banach space $X$. Assume that $A$ is convex. Then $(A, B)$ satisfies the property UC.

After that a weaker notion of property UC was introduced in [3] as follows.

Definition 5. Let $A$ and $B$ be nonempty subsets of a metric space $(X, d)$. The pair $(A, B)$ is said to satisfies the property WUC if for any sequence $\left\{x_{n}\right\}$ in $A$ such that for every $\varepsilon>0$ there exists $y \in B$ satisfying that $d\left(x_{n}, y\right) \leq \operatorname{dist}(A, B)+\varepsilon$ for $n \geq N$, then it is the case that $\left\{x_{n}\right\}$ is convergent. 
It was proved in [3] that if $A$ and $B$ are two nonempty subsets of a complete pointwise uniformly convex geodesic metric space $(X, d)$ with monotone modulus of convexity such that $A$ is convex, then $(A, B)$ satisfies the property WUC (see Proposition 3.15 of [3]).

Here, we recall some notions in partially ordered metric spaces from [5]

Definition 6. Let $(X, \preceq)$ be a partially ordered set. A self mapping $T: X \rightarrow X$ is said to be monotone nondecreasing iff $T(x) \preceq T(y)$ whenever $x, y \in X, x \preceq y$.

Definition 7. Let $(X, \preceq)$ be a partially ordered set and $d$ be a metric on $X$. A subset $A$ of $X$ is said to be a regular set provided that for any nondecreasing sequence $\left\{x_{n}\right\}$ in $A$ so that $x_{n} \rightarrow x \in A$, then $x_{n} \preceq x$ for any $n \in \mathbb{N}$.

The following best proximity point theorem is the main result of [1].

Theorem 3. (Theorem 3.5 of [1]) Let $(X, \preceq)$ be a partially ordered set and $d$ be a metric on $X$. Let $A, B$ be two nonempty subsets of $X$ such that $(A, B)$ satisfies the property UC. Suppose $A$ is complete and is a regular set and let $T: A \cup B \rightarrow A \cup B$ be a cyclic mapping such that $T$ and $T^{2}$ are nondecreasing on $A$. Moreover,

$$
d\left(T \dot{x}, T^{2} x\right) \leq \alpha d(\dot{x}, T x)+(1-\alpha) \operatorname{dist}(A, B),
$$

and

$$
d\left(T \dot{y}, T^{2} y\right) \leq \alpha d(\dot{y}, T y)+(1-\alpha) \operatorname{dist}(A, B),
$$

for some $\alpha \in[0,1[$ and for all $(x, \dot{x}) \in A \times A,(y, \hat{y}) \in B \times B$ with $x \preceq \dot{x}, y \preceq \dot{y}$. If there exists $x_{0} \in A$ such that $x_{0} \preceq T^{2} x_{0}$ and $x_{n+1}=T x_{n}$, then $T$ has a best proximity point $p \in A$ and $x_{2 n} \rightarrow p$.

In the current paper, we prove some existence and convergence results of best proximity points for a new class of mappings, called $F$ - cyclic contractions in the setting on ordered metric spaces. Our conclusions extend and improve some recent results in the literature. We also provide some examples to illustrate our main results.

\section{MAIN RESULTS}

\subsection{Fixed point results}

In this section, we provide some new fixed point results in partially ordered metric spaces for cyclic mappings which satisfy the Wardowski type contraction.

We begin our main results with the following auxiliary lemma.

Lemma 1. Let $(A, B)$ be nonempty pair of subsets of a metric space $(X, d)$ and " $\preceq$ " be a partially ordered relation on $A$ such that $A$ is complete. Let $T: A \cup B \rightarrow$ $A \cup B$ be a cyclic mapping such that $T^{2}$ is nondecreasing on $A$ and there exists $\tau>0$ such that

$$
d^{*}\left(T \dot{x}, T^{2} x\right)>0 \Rightarrow \tau+F\left(d^{*}\left(T \dot{x}, T^{2} x\right) \leq F\left(d^{*}\left(x^{\prime}, T x\right)\right),\right.
$$


for all $x, \dot{x} \in A$ with $x \preceq \dot{x}$. If there exists $x_{0} \in A$ with $x_{0} \preceq T^{2} x_{0}$ and if $x_{n+1}=T x_{n}$, then $d\left(x_{n}, x_{n+1}\right) \rightarrow \operatorname{dist}(A, B)$.

Proof. Since $T^{2}$ is nondecreasing on $A$ and $x_{0} \preceq T^{2} x_{0}$,

$$
x_{0} \preceq T^{2} x_{0} \preceq \cdots \preceq T^{2 n} x_{0} \preceq \cdots .
$$

Put $r_{n}=d^{*}\left(x_{n+1}, x_{n}\right)$. By the fact that $x_{0} \preceq T^{2} x_{0}$,

$$
\begin{aligned}
& F\left(d^{*}\left(x_{3}, x_{2}\right)\right)=F\left(d^{*}\left(T\left(T^{2} x_{0}\right), T^{2} x_{0}\right)\right) \\
\leq & F\left(d^{*}\left(T^{2} x_{0}, T x_{0}\right)\right)-\tau=F\left(d^{*}\left(x_{1}, x_{2}\right)\right)-\tau .
\end{aligned}
$$

Also,

$$
\begin{gathered}
F\left(d^{*}\left(x_{5}, x_{4}\right)\right)=F\left(d^{*}\left(T\left(T^{4} x_{0}\right), T^{2}\left(T^{2} x_{0}\right)\right)\right) \\
\leq F\left(d^{*}\left(T^{4} x_{0}, T^{3} x_{0}\right)\right)-\tau=F\left(d^{*}\left(T\left(T^{2} x_{0}\right), T^{2}\left(T^{2} x_{0}\right)\right)\right)-\tau \\
\leq\left\{F\left(d^{*}\left(T^{2} x_{0}, T^{3} x_{0}\right)\right)-\tau\right\}-\tau=F\left(d^{*}\left(x_{3}, x_{2}\right)\right)-2 \tau \\
\leq F\left(d^{*}\left(x_{2}, x_{1}\right)\right)-3 \tau .
\end{gathered}
$$

Continuing this process and by induction, we obtain

$$
F\left(d^{*}\left(x_{2 n+1}, x_{2 n}\right)\right) \leq F\left(d^{*}\left(x_{2}, x_{1}\right)\right)-(2 n-1) \tau, \quad \forall n \in \mathbb{N},
$$

which deduces that $\lim _{n \rightarrow \infty} F\left(d^{*}\left(x_{2 n+1}, x_{2 n}\right)\right)=-\infty$. From the condition (F2), $d^{*}\left(x_{2 n+1}, x_{2 n}\right) \rightarrow 0$ or $d\left(x_{2 n+1}, x_{2 n}\right) \rightarrow \operatorname{dist}(A, B)$. Similarly, we can see that

$$
F\left(d^{*}\left(x_{2 n+2}, x_{2 n+1}\right)\right) \leq F\left(d^{*}\left(x_{2}, x_{1}\right)\right)-2 n \tau, \quad \forall n \in \mathbb{N},
$$

which implies that $d\left(x_{2 n+1}, x_{2 n+2}\right) \rightarrow \operatorname{dist}(A, B)$. Hence, $d\left(x_{n}, x_{n+1}\right) \rightarrow \operatorname{dist}(A, B)$ and the result follows.

The following fixed point result is motivated by Theorem 2 .

Theorem 4. Let $(A, B)$ be nonempty pair of subsets of a metric space $(X, d)$ and " $\preceq$ " be a partially ordered relation on $A$. Let $T: A \cup B \rightarrow A \cup B$ be a cyclic mapping such $\left.T\right|_{A}$ is continuous and that $T^{2}$ is nondecreasing on $A$ and there exists $\tau>0$ such that

$$
d\left(T \dot{x}, T^{2} x\right)>0 \Rightarrow \tau+F\left(d\left(T \dot{x}, T^{2} x\right) \leq F\left(d\left(x^{\prime}, T x\right)\right),\right.
$$

for all $x, \dot{x} \in A$ with $x \preceq \dot{x}$. If there exists $x_{0} \in A$ with $x_{0} \preceq T^{2} x_{0}$ and if $x_{n+1}=T x_{n}$, then $A \cap B$ is nonempty and $T$ has a fixed point in $A \cap B$. Moreover, $\left\{x_{2 n}\right\}$ converges to the fixed point of $T$.

Proof. We consider the following two cases:

Case 1. $T^{2} x_{0}=x_{0}$. Now if $d\left(x_{0}, T x_{0}\right)>0$, then

$$
\begin{gathered}
F\left(d\left(x_{0}, T x_{0}\right)\right)=F\left(d\left(T^{2} x_{0}, T\left(T^{2} x_{0}\right)\right)\right)=F\left(d\left(T\left(T^{2} x_{0}\right), T^{2} x_{0}\right)\right) \\
\leq F\left(d\left(T^{2} x_{0}, T x_{0}\right)\right)-\tau=F\left(d\left(x_{0}, T x_{0}\right)\right)-\tau<F\left(d\left(x_{0}, T x_{0}\right)\right),
\end{gathered}
$$


which is impossible. That is, $x_{0}=T x_{0}$ and we are finished.

Case 2. $T^{2} x_{0} \neq x_{0}$. Put $r_{n}=d\left(x_{n+1}, x_{n}\right)$. It now follows from a similar argument of Lemma 1 that

$$
F\left(r_{2 n}\right) \leq F\left(r_{0}\right)-2 n \tau, \quad F\left(r_{2 n-1}\right) \leq F\left(r_{1}\right)-2(n-1) \tau .
$$

Hence, $\max \left\{r_{2 n-1}, r_{2 n}\right\} \rightarrow 0$. From (F3) there exist $k_{1}, k_{2} \in(0,1)$ so that

$$
\lim _{n \rightarrow \infty} r_{2 n}^{k_{1}} F\left(r_{2 n}\right)=0, \quad \lim _{n \rightarrow \infty} r_{2 n-1}^{k_{2}} F\left(r_{2 n-1}\right)=0 .
$$

We have

$$
\begin{gathered}
r_{2 n}^{k_{1}} F\left(r_{2 n}\right)-r_{2 n}^{k_{1}} F\left(r_{0}\right) \leq r_{2 n}^{k_{1}}\left(F\left(r_{0}-2 n \tau\right)\right)-r_{2 n}^{k_{1}} F\left(r_{0}\right) \\
=-r_{2 n}^{k_{1}} 2 n \tau \leq 0, \quad \forall n \in \mathbb{N} .
\end{gathered}
$$

Thus $\lim _{n \rightarrow \infty} n r_{2 n}^{k_{1}}=0$. Equivalently, we can see that $\lim _{n \rightarrow \infty} n r_{2 n-1}^{k_{2}}=0$. Let $N \in \mathbb{N}$ be such that $\max \left\{n r_{2 n}^{k_{1}}, n r_{2 n-1}^{k_{2}}\right\} \leq 1$ for all $n \geq N$. Now for all $m>n \geq N$ we have

$$
\begin{gathered}
d\left(x_{2 m}, x_{2 n}\right) \leq \sum_{j=n}^{m+1}\left[d\left(x_{2 j}, x_{2 j+1}\right)+d\left(x_{2 j+1}, x_{2 j+2}\right)\right] \\
=\sum_{j=n}^{m}\left(r_{2 j}+r_{2 j+1}\right) \leq \sum_{j=n}^{\infty}\left(r_{2 j}+r_{2 j+1}\right) \\
\sum_{j=n}^{\infty}\left(\frac{1}{j^{\frac{1}{k_{1}}}}+\frac{1}{j^{\frac{1}{k_{2}}}}\right)<\sum_{j=1}^{\infty}\left(\frac{1}{j^{\frac{1}{k_{1}}}}+\frac{1}{j^{\frac{1}{k_{2}}}}\right)<\infty .
\end{gathered}
$$

Therefore, the sequence $\left\{x_{2 n}\right\}$ is a Cauchy sequence and hence is convergent to a point $p \in A$. In view of the fact that $T$ is continuous on $A, x_{2 n+1}=T x_{2 n} \rightarrow T p$ and so

$$
d(p, T p)=\lim _{n \rightarrow \infty} d\left(x_{2 n}, x_{2 n+1}\right)=\lim _{n \rightarrow \infty} r_{2 n}=0,
$$

that is, $p$ is a fixed point of $T$ in $A \cap B$.

Remark 1. The condition of continuity of the mapping $T$ on $A$ in Theorem 4 can be replaced with the condition of regularity of the set $A$.

Proof. As in the proof of Theorem 4, the nondecreasing sequence $\left\{x_{2 n}\right\}$ converges to a point $p \in A$. Since $A$ is regular, $x_{2 n} \preceq p$ for all $n \in \mathbb{N}$. We now have

$$
\begin{gathered}
F\left(d\left(T p, x_{2 n}\right)\right)=F\left(d\left(T p, T^{2} x_{2 n-2}\right)\right) \leq F\left(d\left(p, x_{2 n-1}\right)\right)-\tau \\
\quad \leq F\left(d\left(p, x_{2 n}\right), d\left(x_{2 n}, x_{2 n-1}\right)\right)-\tau \rightarrow-\infty \quad(n \rightarrow \infty),
\end{gathered}
$$

and this concludes that $\lim _{n \rightarrow \infty} d\left(T p, x_{2 n}\right)=0$ by (F2) and so, $p=T p$. 
Next corollaries are the straightforward consequences of Theorem 4.

Corollary 1 (Theorems 2.2, 2.3 of [1]). Let $(A, B)$ be a nonempty pair of subsets of a metric space $(X, d)$, such that $A$ is complete, and let " $\leq$ " be a partially ordered relation on $A$. Let $T: A \cup B \rightarrow A \cup B$ be a cyclic mapping and $T^{2}$ be nondecreasing on $A$ and $d\left(T^{x}, T^{2} x\right) \leq \alpha d(\dot{x}, T x)$ for some $\alpha \in[0,1[$ and for all $(x, \dot{x}) \in A \times A$ with $x \preceq \dot{x}$. Assume that $\left.T\right|_{A}$ is continuous or $A$ is a regular set. If there exists $x_{0} \in A$ with $x_{0} \preceq T^{2} x_{0}$, then $A \cap B \neq \varnothing$, hence $T$ has a fixed point $p \in A \cap B$. Moreover, if $x_{n+1}:=T x_{n}$, then $x_{2 n} \rightarrow p$.

Corollary 2 ([5]). Let $(X, \preceq)$ be a partially ordered set and let there exists a metric $d$ in $X$ which makes $(X, d)$ into a complete metric space. Suppose $T: X \rightarrow X$ is a nondecreasing mapping on $X$ such that either $T$ is continuous or $X$ is a regular set. Assume there exists $\alpha \in[0,1[$ such that $d(T x, T y) \leq \alpha d(x, y)$ for each $y \preceq x$. If there exists $x_{0} \in X$ with $x_{0} \preceq T\left(x_{0}\right)$, then $T$ has a fixed point.

The following example illustrates Theorem 4.

Example 1 . Let $(X, \preceq)$ be a partially ordered, where $X=[0,3]$ is complete metric space with metric $d: X \times X \rightarrow[0, \infty)$ defined as $d(x, y)=|x-y|, x, y \in X$, and $x \preceq y \Leftrightarrow x \leqslant y$, for each $x, y \in X$. Let $A=[0,1] \cup[2,3] \subset X$, and $B=(1,3] \subset X$. Of course $\preceq$ is a partially ordered relation on $A$. Let $T: A \cup B \rightarrow A \cup B$ be defined as follows

$$
T(x)= \begin{cases}\frac{15}{8} & \text { if } x \in[0,1] \\ 2 & \text { if } x \in(1,3] .\end{cases}
$$

Of course $\left.T\right|_{A}$ is continuous, and iteration $T^{2}$ is nondecreasing on $A$.

Let $F: \mathbb{R}_{+} \rightarrow \mathbb{R}$ be given by the formula $F(t)=\ln \left(t^{2}+t\right)$, where $t>0$. It is easy to see, that the map $F$ satisfies the conditions (F1)-(F3).

We show that $T$ satisfies the condition (2.1). Let $\tau=\ln (2)>0$. Let $x, \dot{x} \in A$ with $x \preceq x$ (i.e. $x \leqslant x$ ). We consider the following three cases:

Case 1. If $x, x \in[0,1]$, then, by (2.2), we obtain $T(x)=T(x)=\frac{15}{8}, T^{2}(x)=$ $T^{2}(\dot{x})=2$. Hence $d\left(T(\dot{x}), T^{2}(x)\right)=d\left(\frac{15}{8}, 2\right)=\frac{1}{8}>0$. Moreover, since $x \leqslant 1$, by simply calculations, we have $\frac{15}{8}-\dot{x} \geqslant \frac{7}{8}$

$$
\begin{gathered}
{\left[d\left(T(\dot{x}), T^{2}(x)\right)\right]^{2}+d\left(T(\dot{x}), T^{2}(x)\right)=\frac{1}{64}+\frac{1}{8}=\frac{9}{64}} \\
\leqslant \frac{1}{2}\left[\frac{49}{64}+\frac{56}{64}\right] \leqslant \frac{1}{2}\left[\left(\frac{15}{8}-\dot{x}\right)^{2}+\left(\frac{15}{8}-\dot{x}\right)\right]=\frac{1}{2}\left([d(\dot{x}, T(x))]^{2}+d(\dot{x}, T(x))\right) \\
\leqslant\left([d(\dot{x}, T(x))]^{2}+d(\dot{x}, T(x))\right) e^{-\ln (2)} .
\end{gathered}
$$

In consequence,

$$
\left[d\left(T(\dot{x}), T^{2}(x)\right)\right]^{2}+d\left(T(\dot{x}), T^{2}(x)\right) \leqslant\left([d(\dot{x}, T(x))]^{2}+d(\dot{x}, T(x))\right) e^{-\ln (2)} .
$$


Hence,

$$
e^{\ln \left(\left[d\left(T(\dot{x}), T^{2}(x)\right)\right]^{2}+d\left(T(\dot{x}), T^{2}(x)\right)\right)} \leqslant e^{\ln \left([d(\dot{x}, T(x))]^{2}+d(\dot{x}, T(x))\right)} e^{-\ln (2)}
$$

and next

$$
e^{F\left(d\left(T(\dot{x}), T^{2}(x)\right)\right)} \leqslant e^{F(d(\dot{x}, T(x)))} e^{-\ln (2)}=e^{F(d(\dot{x}, T(x)))-\ln (2)}
$$

Finally, we obtain

$$
F\left(d\left(T(\dot{x}), T^{2}(x)\right)\right) \leqslant F(d(\dot{x}, T(x)))-\ln (2)
$$

thus

$$
\tau+F\left(d\left(T(\dot{x}), T^{2}(x)\right)\right) \leqslant F(d(\dot{x}, T(x)))
$$

Case 2. If $x \in[0,1]$ and $x \in[2,3]$, then, by (2.2), we obtain $T(x)=\frac{15}{8}, T(\dot{x})=$ $T^{2}(x)=T^{2}(\dot{x})=2$. Hence $d\left(T(x), T^{2}(x)\right)=d(2,2)=0$.

Case 3. If $x, \dot{x} \in[2,3]$, then, by (2.2), we obtain $T(x)=T(\dot{x})=T^{2}(x)=$ $T^{2}(\dot{x})=2$. Hence $d\left(T(x), T^{2}(x)\right)=d(2,2)=0$.

In consequence of considerations of each cases, we obtain that $T$ satisfies the condition (2.1).

Of course, there exists $x_{0} \in A$ (for example $x_{0}=1$ ) such that $x_{0} \leqslant T^{2}\left(x_{0}\right)$. All assumption of Theorem 2.2 are satisfied. There exists a fixed point $w=2$ of the map $T$ in $A$. Moreover if we define the sequence $\left(x_{n}: n \in \mathbb{N}\right)$ as $x_{n+1}=T\left(x_{n}\right), n \in \mathbb{N}$, then $\lim _{n \rightarrow \infty} x_{2 n}=w$.

\subsection{Best proximity point results}

Motivated by Theorem 3, we establish an existence result of best proximity points for $F$ - cyclic contractions under weaker assumptions.

We begin our main conclusions of this section with the following theorem.

Theorem 5. Let $(A, B)$ be nonempty pair of subsets of a metric space $(X, d)$ and " $\preceq$ " be a partially ordered relation on $A$ such that $A$ is complete. Let $T: A \cup B \rightarrow$ $A \cup B$ be a cyclic mapping such that $T^{2}$ is nondecreasing on $A$ and there exists $\tau>0$ such that

$$
d^{*}\left(T \dot{x}, T^{2} x\right)>0 \Rightarrow \tau+F\left(d^{*}\left(T \dot{x}, T^{2} x\right) \leq F\left(d^{*}\left(x^{\prime}, T x\right)\right),\right.
$$

for all $x, \dot{x} \in A$ with $x \preceq \dot{x}$. Suppose there exists $x_{0} \in A$ with $x_{0} \preceq T^{2} x_{0}$ and define $x_{n+1}=T x_{n}$. If $A$ is a regular set and boundedly compact, then $T$ has a best proximity point.

Proof. Since $T^{2}$ is nondecreasing, the sequence $\left\{x_{2 n}\right\}$ is increasing and by the fact that $A$ is boundedly compact, there exists a subsequence $\left\{x_{2 n_{k}}\right\}$ of $\left\{x_{2 n}\right\}$ converging to some $p \in A$. Thus

$$
\operatorname{dist}(A, B) \leq d\left(p, x_{2 n_{k}-1}\right) \leq d\left(p, x_{2 n_{k}}\right)+d\left(x_{2 n_{k}}, x_{2 n_{k}-1}\right) .
$$


Now if $k \rightarrow \infty$, then by Lemma 1 we have $d\left(p, x_{2 n_{k}-1}\right) \rightarrow \operatorname{dist}(A, B)$. In view of the fact that $A$ is a regular set, $x_{2 n_{k}} \preceq p$ for all $k \in \mathbb{N}$. We have

$$
\begin{gathered}
F\left(d^{*}\left(x_{2 n_{k}}, T p\right)\right)=F\left(d^{*}\left(T p, T^{2} x_{2 n_{k}-2}\right)\right) \\
\leq F\left(d^{*}\left(p, T x_{2 n_{k}-2}\right)\right)-\tau=F\left(d^{*}\left(p, x_{2 n_{k}-1}\right)\right)-\tau \rightarrow-\infty \quad(k \rightarrow \infty) .
\end{gathered}
$$

Therefore, $d^{*}\left(x_{2 n_{k}}, T p\right) \rightarrow 0$ and so $d(p, T p)=\operatorname{dist}(A, B)$.

Remark 2. It is worth noticing that if in Theorem $5 \mathcal{P}_{A}(y)$ is singleton for any $y \in B_{0}$, then $T^{2} p=p$, that is, $p$ is a fixed point of $T^{2}$.

Proof. Since $d(p, T p)=\operatorname{dist}(A, B)$, we have $\mathcal{P}_{A}(T p)=\{p\}$. If $d^{*}\left(T p, T^{2} p\right)>$ 0 , then

$$
F\left(d^{*}\left(T p, T^{2} p\right)\right) \leq F\left(d^{*}(p, T p)\right)-\tau,
$$

which is impossible by the fact that $F \in \mathscr{F}$ and so the domain of $F$ is $(0, \infty)$. Thereby, $d\left(T p, T^{2} p\right)=\operatorname{dist}(A, B)$ and so $T^{2} p \in \mathcal{P}_{A}(T p)$ and this turns that $T^{2} p=$ $p$.

Definition 8. Let $A$ and $B$ be nonempty subsets of a partially metric space $(X, d)$. The pair $(A, B)$ is said to have monotone proximally property if for any increasing sequence $\left\{x_{n}\right\}$ in $A$ such that for every $\varepsilon>0$ there exist $y \in B$ and $N \in \mathbb{N}$ satisfying that $d\left(x_{n}, y\right) \leq \operatorname{dist}(A, B)+\varepsilon$ for $n \geq N$, then it is the case that $\left\{x_{n}\right\}$ has a convergent subsequence.

Next example shows that the monotone proximally property does not imply property WUC in general.

Example 2.2. Consider $X=\mathbb{R}$ with the usual metric and with the natural partially ordered relation $\leq$. Suppose $A=(-\infty, 0] \cup\{2\}$ and $B=\{1\}$. Then $\operatorname{dist}(A, B)=1$ and $A_{0}=\{0,2\}, B_{0}=\{1\}$. Let $\varepsilon>0$ be given. We have the following two cases:

Case 1. Consider the nondecreasing sequence $\left\{x_{n}\right\}$ defined with $x_{n}=-\frac{1}{n}$. Then for $y=1$ we have $\lim _{n \rightarrow \infty} d\left(x_{n}, y\right)=1$ and so, there exists $N \in \mathbb{N}$ such that $d\left(x_{n}, y\right) \leq \operatorname{dist}(A, B)+\varepsilon$ for all $n \geq N$. We note that $x_{n} \rightarrow 0$.

Case 2. Let $\left\{x_{n}\right\}$ be a sequence in $A$ for which $x_{n}=2$ for all $n \in \mathbb{N}$ except perhaps finite numbers. In this case we have $x_{n} \rightarrow 2$ and that $\lim _{n \rightarrow \infty} d\left(x_{n}, y\right)=1$.

Therefore, $(A, B)$ has the monotone proximally property.

We now claim that $(A, B)$ does not satisfy the property WUC. To this end, define the sequence $\left\{z_{n}\right\}$ in $A$ as

$$
z_{n}=\left\{\begin{array}{l}
-\frac{1}{n} \text { if } n \text { is odd } \\
2 \text { if } n \text { is even }
\end{array}\right.
$$


The we have $\lim _{n \rightarrow \infty} d\left(z_{n}, y\right)=\operatorname{dist}(A, B)$ but the sequence $\left\{z_{n}\right\}$ is not convergent.

Example 2.3. Suppose $X=\mathbb{R}^{2}$ and define the metric $d$ on $X$ by

$$
d\left(\left(x_{1}, y_{1}\right),\left(x_{2}, y_{2}\right)\right)=\left|x_{1}-x_{2}\right|+\left|y_{1}-y_{2}\right|, \quad \forall\left(x_{1}, y_{1}\right),\left(x_{2}, y_{2}\right) \in \mathbb{R}^{2} .
$$

Consider the partially ordered relation on $X$ with $\left(x_{1}, y_{1}\right) \preceq\left(x_{2}, y_{2}\right) \Leftrightarrow x_{1} \leq x_{2}, y_{1} \leq$ $y_{2}$. We define

$$
A=\left\{\left(0,1-\frac{1}{2 n}\right): n \in \mathbb{N}\right\} \cup\{(0, n): n \in \mathbb{N}\}, \quad B=\{(2, y): 0 \leq y \leq 1\} .
$$

By simply calculations, we obtain $\operatorname{dist}(A, B)=2$. By definition of $\mathrm{A}$, we see that there exists only one increasing sequence $\left\{x_{n}\right\}$ in $A$ such that for every $\varepsilon>0$ there exist $y \in B$ and $N \in \mathbb{N}$ satisfying that $d\left(x_{n}, y\right) \leq \operatorname{dist}(A, B)+\varepsilon$ for $n \geq N$. The above assumption satisfies the sequence $\left\{x_{n}=\left(0,1-\frac{1}{2 n}\right): n \in \mathbb{N}\right\}$ for $y=(2,1)$. Then, of course $\left\{x_{n}\right\}$ has a convergent subsequence (for example $\left\{x_{k}=\left(0,1-\frac{1}{4 k}\right): k \in \mathbb{N}\right\}$ ).

The following theorem is the main result of this section.

Theorem 6. Let $(A, B)$ be nonempty pair of subsets of a metric space $(X, d)$ and " $\preceq$ " be a partially ordered relation on $A$ such that $A$ is complete. Let $T: A \cup B \rightarrow$ $A \cup B$ be a cyclic mapping such that $T^{2}$ is nondecreasing on $A$ and there exists $\tau>0$ such that

$$
d^{*}\left(T \dot{x}, T^{2} x\right)>0 \Rightarrow \tau+F\left(d^{*}\left(T \dot{x}, T^{2} x\right) \leq F\left(d^{*}\left(x^{\prime}, T x\right)\right),\right.
$$

for all $x, \dot{x} \in A$ with $x \preceq \dot{x}$. Suppose there exists $x_{0} \in A$ with $x_{0} \preceq T^{2} x_{0}$ and define $x_{n+1}=T x_{n}$. If $A$ is bounded and a regular set and $(A, B)$ has the monotone proximally property, then $T$ has a best proximity point.

Proof. From Lemma $1 d\left(x_{n}, x_{n+1}\right) \rightarrow \operatorname{dist}(A, B)$. Since $A$ is bounded, the sequence $\left\{x_{2 n}\right\}$ and so the sequence $\left\{x_{2 n-1}\right\}$ are also bounded. Consider $n \in \mathbb{N}$. Then for all $k \in \mathbb{N}$ by the fact that the sequences $\left\{x_{2 n-1}\right\}$ and $\left\{x_{2 n}\right\}$ are nondecreasing and bounded, we have

$$
\begin{gathered}
F\left(d^{*}\left(x_{2 n+2 k}, x_{2 n+1}\right)\right) \\
=F\left(d^{*}\left(T^{2 n+2 k} x_{0}, T^{2 n+1} x_{0}\right)\right)=F\left(d^{*}\left(T\left(x_{2 n+2 k-1}\right), T^{2}\left(x_{2 n-1}\right)\right)\right) \\
\leq F\left(d^{*}\left(x_{2 n+2 k-1}, x_{2 n}\right)\right)-\tau \quad\left(\text { since } x_{2 n-1} \preceq x_{2 n+2 k-1}\right) \\
=F\left(d^{*}\left(T x_{2 n+2 k-2}, T^{2} x_{2 n-2}\right)\right)-\tau \\
\leq F\left(d^{*}\left(x_{2 n+2 k-2}, x_{2 n-1}\right)\right)-2 \tau \quad\left(\text { since } x_{2 n-2} \preceq x_{2 n+2 k-2}\right) \\
\leq \ldots \leq F\left(d^{*}\left(x_{2 k}, x_{1}\right)\right)-2 n \tau \leq F(M)-2 n \tau,
\end{gathered}
$$

where $M:=\sup \left\{d^{*}\left(x_{2 k}, x_{1}\right): k \in \mathbb{N}\right\}$. Thus for all $\varepsilon>0$ there exists $n_{0} \in \mathbb{N}$ such that $d^{*}\left(x_{2 n_{0}+2 k}, x_{2 n_{0}+1}\right) \leq \varepsilon$ which implies that

$$
x_{2 n_{0}+2 k} \in \mathscr{B}\left(x_{2 n_{0}+1}, \varepsilon+\operatorname{dist}(A, B)\right), \quad \forall k \in \mathbb{N} .
$$


By the fact that $(A, B)$ has the monotone proximally property, we conclude that the sequence $\left\{x_{2 n}\right\}$ has a convergent subsequence to a point $p \in A$. It now follows from an equivalent argument of Theorem 5 that $p$ is a best proximity point of $T$ and the result follows.

Let us illustrate Theorem 6 with the following example.

Example 2.4. Suppose $X=\mathbb{R}^{2}$ and define the metric $d$ on $X$ by

$$
d\left(\left(x_{1}, y_{1}\right),\left(x_{2}, y_{2}\right)\right)=\left|x_{1}-x_{2}\right|+\left|y_{1}-y_{2}\right|, \quad \forall\left(x_{1}, y_{1}\right),\left(x_{2}, y_{2}\right) \in \mathbb{R}^{2} .
$$

We know that $X$ is not strictly convex. Consider the partially ordered relation on $X$ with

$$
\left(x_{1}, y_{1}\right) \preceq\left(x_{2}, y_{2}\right) \Leftrightarrow x_{1} \leq x_{2}, y_{1} \leq y_{2} .
$$

Let

$$
A=\left\{\left(0,1-\frac{1}{2 n}\right): n \in \mathbb{N}\right\} \cup\{(0, n): n \in \mathbb{N}\}, \quad B=\{(2, y): 0 \leq y \leq 1\} .
$$

Then $\operatorname{dist}(A, B)=2$ and $A_{0}=\left\{\left(0,1-\frac{1}{2 n}\right): n \in \mathbb{N}\right\} \cup\{(0, n): n \in\{1,2,3\}\}, B_{0}=B$. Also, it is easy to see that $(A, B)$ has the monotone proximally property. Define the cyclic mapping $T: A \cup B \rightarrow A \cup B$ by formula

$$
T(x)=
$$

$(2,1)$ for $x \in W:=\left\{\left(x_{1}, y_{1}\right) \in X: x_{1}=0 \wedge y_{1} \in\{1,2\} \cup\left\{1-\frac{1}{2 n}: n \in \mathbb{N}\right\}\right\}$

$(2,0)$ for $x \in V:=\left\{\left(x_{1}, y_{1}\right) \in X: x_{1}=0 \wedge y_{1} \in\{n \in \mathbb{N}: n>2\}\right\}$

$(0,1)$ for $x=\left(x_{1}, y_{1}\right)=(2, k), k \in[0,1]$

where $x \in A \cup B$. Then, we calculate

$$
T^{2}(x)=\left\{\begin{array}{ll}
(0,1) & \text { for } x \in W \cup V \\
(2,1) & \text { for } x=\left(x_{1}, y_{1}\right)=(2, k), k \in[0,1]
\end{array}, x \in A \cup B .\right.
$$

It is easy to observe that the map $T$ is continuous on $A$, and $T^{2}$ is nondecreasing on A.

Next, we show that, for $F(t)=\ln (t), t>0$, there exists $\tau>0$ such that

$$
d^{*}\left(T \dot{x}, T^{2} x\right)>0 \Rightarrow \tau+F\left(d^{*}\left(T \dot{x}, T^{2} x\right) \leq F\left(d^{*}\left(x^{\prime}, T x\right)\right),\right.
$$

for all $x, \dot{x} \in A$ with $x \preceq x$.

Indeed, let $x, \dot{x} \in A$ with $x \preceq \dot{x}$ and $\tau=\ln (2)>0$. Since $x \preceq \dot{x}$, we may consider three cases:

Case 1. If $x, \dot{x} \in W$, then we have the following: $T(x)=(2,1), T^{2}(x)=(0,1)$, $T(\hat{x})=(2,1), T^{2}(\hat{x})=(0,1)$, thus $d\left(T(\hat{x}), T^{2}(x)\right)=d((2,1),(0,1))=2$, and $d^{*}\left(T(\hat{x}), T^{2}(x)\right)=d\left(T(\hat{x}), T^{2}(x)\right)-\operatorname{dist}(A, B)=0$. In consequence the contraction conditions is trivial satisfies. 
Case 2. If $x, \dot{x} \in V$, then we have the following: $T(x)=(2,0), T^{2}(x)=(0,1)$, $T(\dot{x})=(2,0), T^{2}(\dot{x})=(0,1)$, thus $d\left(T(\dot{x}), T^{2}(x)\right)=d((2,0),(0,1))=3$, and $d^{*}\left(T(\dot{x}), T^{2}(x)\right)$

$=d\left(T(\hat{x}), T^{2}(x)\right)-\operatorname{dist}(A, B)=1>0$. Moreover, $d(\dot{x}, T(x))=d((0, m),(2,0))=$ $m+2$, and $d^{*}(\dot{x}, T(x))=d(\dot{x}, T(x))-\operatorname{dist}(A, B)=m$, for some $m>2$, such that $\dot{x}=(0, m) \in V$. On the other hand, we have $m \geq 3$, so $\frac{1}{m} \leq \frac{1}{3}<\frac{1}{2}$. In consequence, since $\frac{1}{2}=e^{-\ln (2)}=e^{-\tau}$, we obtain

$$
d^{*}\left(T(\dot{x}), T^{2}(x)\right)=1 \leq m e^{-\tau}=d^{*}(\dot{x}, T(x)) e^{-\tau} .
$$

Therefore

$$
e^{\tau} d^{*}\left(T(\dot{x}), T^{2}(x)\right) \leq d^{*}(\dot{x}, T(x))
$$

and next

$$
e^{\tau} e^{\ln \left(d^{*}\left(T(\hat{x}), T^{2}(x)\right)\right)} \leq e^{\ln \left(d^{*}(\dot{x}, T(x))\right)} .
$$

Finally, we obtain

$$
\tau+F\left(d^{*}\left(T(\dot{x}), T^{2}(x)\right)\right) \leq F\left(d^{*}(\dot{x}, T(x))\right) .
$$

Hence, the contraction conditions is satisfies in this case.

Case 3. If $x \in W$ and $x \in V$, then we have the following: $T(x)=(2,1), T^{2}(x)=$ $(0,1), T(\hat{x})=(2,0), T^{2}(\dot{x})=(0,1)$, thus $d\left(T(\hat{x}), T^{2}(x)\right)=d((2,0),(0,1))=3$, and $d^{*}\left(T(\hat{x}), T^{2}(x)\right)=d\left(T(\hat{x}), T^{2}(x)\right)-\operatorname{dist}(A, B)=1>0$. Moreover,

$d(\dot{x}, T(x))=d((0, m),(2,1))=m+1$, and $d^{*}(\dot{x}, T(x))=d(\dot{x}, T(x))-\operatorname{dist}(A, B)$ $=m-1$, for some $m>2$, such that $\dot{x}=(0, m) \in V$. On the other hand, we have $m \geq 3$, so $m-1 \geq 2$ and next $1 \leq \frac{1}{2}(m-1)$. In consequence, since $\frac{1}{2}=e^{-\tau}$, we obtain

$$
d^{*}\left(T(\dot{x}), T^{2}(x)\right)=1 \leq(m-1) e^{-\tau}=d^{*}(\dot{x}, T(x)) e^{-\tau} .
$$

Therefore

$$
e^{\tau} d^{*}\left(T(\dot{x}), T^{2}(x)\right) \leq d^{*}(\dot{x}, T(x)),
$$

and next

$$
e^{\tau} e^{\ln \left(d^{*}\left(T(\dot{x}), T^{2}(x)\right)\right)} \leq e^{\ln \left(d^{*}(\dot{x}, T(x))\right)} .
$$

Finally, we obtain

$$
\tau+F\left(d^{*}\left(T(\dot{x}), T^{2}(x)\right)\right) \leq F\left(d^{*}(\dot{x}, T(x))\right) .
$$

Hence, the contraction conditions is satisfies in this case.

In consequence, for the map $T$ there exists $\tau>0$ such that

$$
d^{*}\left(T \dot{x}, T^{2} x\right)>0 \Rightarrow \tau+F\left(d^{*}\left(T \dot{x}, T^{2} x\right) \leq F\left(d^{*}\left(x^{\prime}, T x\right)\right),\right.
$$

for all $x, \dot{x} \in A$ with $x \preceq \dot{x}$. We observe that exists $x_{0} \in A$ with $x_{0} \preceq T^{2} x_{0}$ (for example $x_{0}=\left(0, \frac{1}{2}\right)$ and then $\left.T^{2} x_{0}=(0,1)\right)$. Ofcourse, the set $A$ is bounded and a regular set and $(A, B)$ has the monotone proximally property (see Example 3.2). Finally, the point $(0,1)$ is the best proximity point of $T$ (we recall $T(0,1)=(2,1)$, 
so $d((0,1), T(0,1))=2=\operatorname{dist}(A, B))$.

\section{ACKNOWLEDGEMENT}

The authors declare that they have no competing interests. All authors contributed equally and significantly in writing this article. All authors read and approved the final manuscript.

\section{REFERENCES}

[1] A. Abkar and M. Gabeleh, "Best proximity points for cyclic mappings in ordered metric spaces." J. Optim. Theory Appl., no. 150, pp. 188-193, 2011.

[2] A. Eldred and P. A., Veeramani, "Existence and convergence of best proximity points," J. Math. Anal. Appl., no. 323, pp. 1001-1006, 2006.

[3] R. Espninola and A. Fernaandez-Leaon, "On best proximity points in metric and Banach spaces," Canad. Math. Bull., no. 63, pp. 533-550, 2011.

[4] W. Kirk, P. Srinivasan, and P. Veeramani, "Fixed points for mappings satisfying cyclic contractive conditions," Fixed Point Theory, vol. 4, no. 1, pp. 79-86, 2003.

[5] J. Nieto and R. Rodri'guez-Lo'pez, "Contractive mapping theorems in partially ordered sets and applications to ordinary diferential equations," Order, no. 22, pp. 223-239, 2005.

[6] T. Suzuki, M. Kikkawa, and C. Vetro, "The existence of best proximity points in metric spaces with the property UC," Nonlinear Analysis, no. 71, pp. 2918-2926, 2009.

[7] D. Wardowski, "Fixed point of a new type of contractive mappings in complete metric spaces," Fixed Point Theory Appl., no. doi:10.1186/1687-1812-2012-94, pp. 1-6, 2012.

Authors' addresses

M. Gabeleh

Department of Mathematics, Ayatollah Boroujerdi, Boroujerd, Iran

E-mail address: gab.moo@gmail.com

O. Olela Otafudu

School of Mathematics University of the Witwatersrand, 2050 Johannesburg, South Africa

E-mail address: olmaolela@gmail.com

R. Plebaniak

University of Lodz, Department of Nonlinear Analysis, Banacha 22, 90-238 Lodz, Poland

E-mail address: robpleb@math.uni.lodz.pl 